\title{
The Potential of Light Microscopic Features of the Oral Mucosa in Predicting Post-mortem Interval
}

$$
\text { إحتمالية الاستدلال بالتغيرات المجهرية لغشاء الفم المخاطي للتنبؤ عن مرحلة ما بعد الوفاة }
$$

جاغاناث باترو، سواجاتيكا باندا، نيتا موهـانتي، أوما سانكار ميشرا

ABSTRACT: Objectives: The post-mortem interval (PMI) refers to the amount of time elapsed between death and discovery of the body. This study aimed to evaluate light microscopic cellular changes in the oral mucosa and identify the potential of this method for predicting PMI. Methods: This prospective study was conducted between July 2016 and January 2018 at the Institute of Dental Sciences, Siksha 'O' Anusandhan University, Bhubaneswar, India. A total of 150 post-mortem (including 75 gingival and 75 buccal mucosa samples) and 40 ante-mortem (including 20 gingival and 20 buccal mucosa samples) tissue samples were compared using haematoxylin and eosin, periodic acid-Schiff (PAS) and van Gieson stains. Microscopic changes in the epithelium and connective tissue were categorised according to PMI stage as early ( $<12.5$ hours since death), intermediate (12.5-20.5 hours since death) or late (>20.5 hours since death). Results: Most epithelial cellular changes occurred early, except for arc-shaped nuclei and epithelial shredding which were intermediate and late changes, respectively. However, microscopic changes in the connective tissue were only observable at $\geq 12.5$ hours. There was a progressive decrease in intensity in van Gieson stains and an increase in intensity in PAS stains as PMI increased. Several microscopic features were found to be significant predictors of PMI including epithelial homogenisation, cytoplasmic vacuolation, nuclear degeneration, arc-shaped nuclei, chromatin clumping, red blood cell clumping and lysis, melanin incontinency, myofibril degeneration, salivary gland acini degeneration and epithelial connective tissue separation $(P<0.050$ each). Conclusion: These findings indicate that microscopic evaluation of the oral mucosa may be helpful for PMI prediction.

Keywords: Post-mortem Changes; Light Microscopy; Oral Mucosa; Epithelial Cells; Lamina Propria; Salivary Glands; Histocytochemistry; Periodic Acid-Schiff Reaction; India.

الملخص: الهدف: تشير فترة ما بعد الوفاة (PMI) إلى مقدار الوقت المنقضي بين الموت واكتشاف الجسد. هدفت هذه الدراسة إلى تقييم

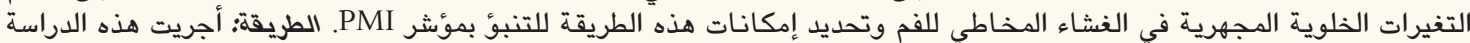

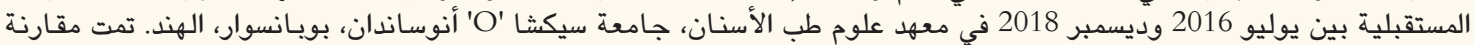

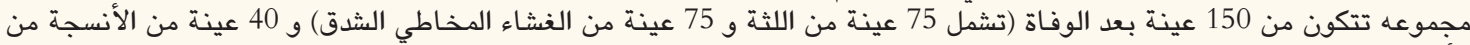

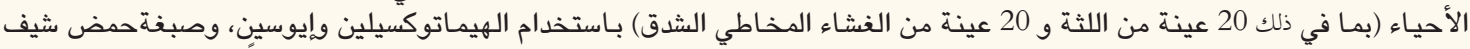

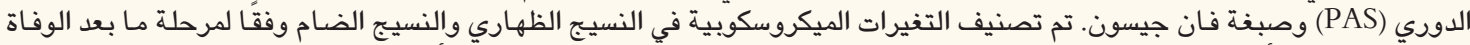

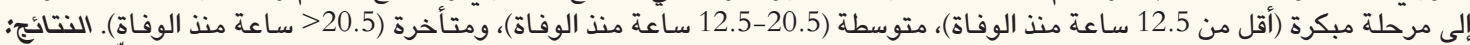

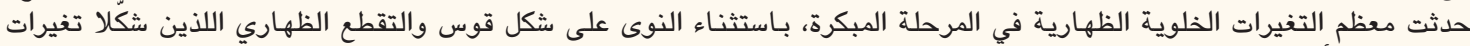

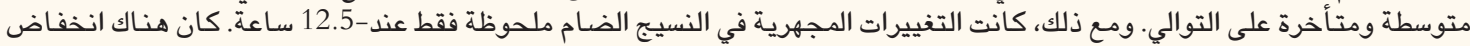

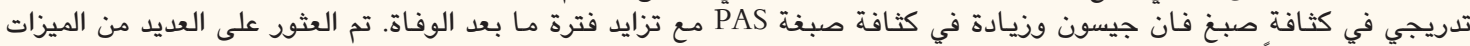

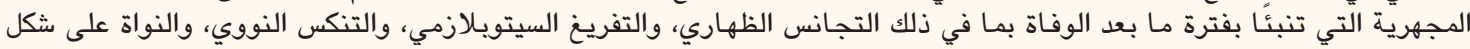

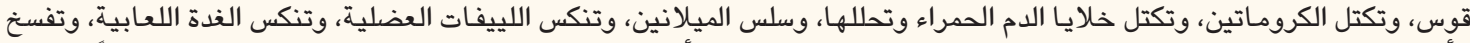

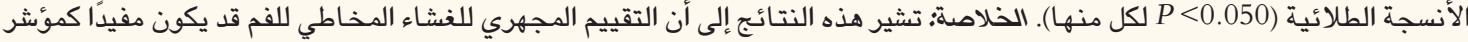

$$
\text { للتنبؤ عن فترة ما بعد الوفاة }
$$

الكلمات المفتاحية: تغييرات ما بعد الوفاة؛ المجهر الضوئي؛ الغشاء الفئاء المخاطي للفم الخلايا الظهارية؛ الغشاء القاعدي؛ الغدد اللعابية؛ كيمياء الأنسجة؛ تفاعل حمض شيف الدوري؛ الهند.

\section{AdVANCES IN KNOWLEDGE}

The findings of the study not only emphasise the potential of light microscopy in the estimation of post-mortem interval, but also highlight the importance of special stains other than haematoxylin and eosin as a complementary tool in forensic investigation.

To the best of the authors' knowledge, this study is the first of its kind to depict the sequential, temporal, cellular and architectural histological features of the oral mucosa after death in a large sample using two distinct kinds of mucosal samples and three staining techniques. 


\section{Application to Patient Care}

Estimation of time since death is an important forensic tool in identifying human remains and determining possible causes of death, thereby indirectly influencing patient care.

\section{$\mathrm{T}$} HANATOLOGY REFERS TO THE PHILOSOPHICAL and scientific study of various aspects of death including the forensic examination of bodily changes which take place at death and during the post-mortem period. While dozens of methods are currently used to determine the amount of time elapsed between death and discovery of the body-a period known as the post-mortem interval (PMI) there is nevertheless still demand for more reliable and precise techniques. ${ }^{1}$ Currently, many approaches to PMI estimation focus on observable and measurable changes to the body after death such as autolysis, algor mortis, rigor mortis, livor mortis and the digestive status of the stomach contents. ${ }^{1,2}$

Following death, many physical and chemical changes begin to take place and continue until the body eventually decomposes. At the onset of death, respiration ceases and cell glycolysis begins, leading to the production of lactic acid followed by a drop in the $\mathrm{pH}$ of the cellular contents. ${ }^{3}$ Eventually, cellular metabolism comes to an end and the subsequent predominance of lytic enzymes results in autolysis. The cell membranes disintegrate and a reduction in adenosine triphosphate levels causes loss of the cellular architecture and fatty acids. ${ }^{3}$ Cell swelling occurs due to a decrease in energy level; subsequently, the cells progressively detach from one another in a process known as necrosis. The reduction in cellular energy causes disruption of the mitochondrial electron transport system and leads to fermentation, resulting in the formation of anaerobic lactic acid from pyruvic acid. ${ }^{3}$

Utilising light microscopy, cells are perceived as dead after they have undergone a series of specific morphological changes which occur as a result of two distinct cellular processes: (1) apoptosis, a programmed and orderly form of cell death and (2) necrosis, a disorderly and unpredictable form of cell death. Necrotic cells show an increased eosinophilia. Nucleus of the epithelial cells may undergo changes like karyolysis, pyknosis and karyorrhexis. ${ }^{4}$

Post-mortem cellular changes have been proven to reliably estimate PMI in several organs including the liver, kidney, pancreas and heart., ${ }^{5,6}$ As in other tissues, autolytic enzymes are released by the oral mucosal cells after death, resulting in abnormal morphology. Therefore, researchers have explored the possibility of identifying time since death via microscopic examination of the oral mucosa. ${ }^{7}$ Degenerative post-mortem changes to the epithelium and connective tissue in the oral mucosa may demonstrate specific patterns that could prove useful to determine PMI; however, few studies have sought to evaluate post-mortem cellular changes in the oral tissues including the gingiva, salivary glands and dental pulp. ${ }^{7-14}$ Moreover, inadequacies in terms of sample size and standardised methodology have often precluded confirmation of this approach. Therefore, the current study aimed to investigate post-mortem light microscopic cellular changes in the oral tissues with the objective of estimating their potential for PMI prediction.

\section{Methods}

This prospective study took place over an 18-month period between July 2016 and January 2018 at the Institute of Dental Sciences, Siksha 'O' Anusandhan University, Bhubaneswar, Odisha, India, in collaboration with two government medical colleges in Odisha. Oral mucosa samples were collected from the bodies of recently deceased individuals aged $18-45$ years old. In each case, information was obtained concerning cause of death, age, gender, time and date of death and time spent outside or in a natural environment since death. The bodies of individuals with a history of chronic generalised periodontitis, neoplastic growth or communicable disease were excluded, as were those of pregnant females, victims with facial burns and those who had been preserved after death.

The bodies were placed in a dry environment with adequate light and kept at room temperature. Oral tissues were collected under the direct supervision of a forensic medicine and toxicology specialist. The gingival and buccal mucosa were accessed using basic diagnostic instruments including a mouth mirror, a no. 5 interproximal explorer, a pair of tweezers and a pair of toothed forceps. Samples were collected using a round punch knife of $6 \mathrm{~mm}$ in diameter, a no. 15 Bard-Parker ${ }^{\circledR}$ stainless steel blade (Aspen Surgical Corp., Caledonia, Michigan, USA) and a Langenbeck retractor to retract the buccal mucosa. A total of 150 mucosa samples of approximately $6 \times 8 \times 5 \mathrm{~mm}$ in size were collected, comprising both gingival $(n=75)$ and buccal $(n=75)$ samples. In addition, 40 antemortem samples of keratinised $(n=20)$ and non-keratinised $(n$ $=20$ ) oral mucosa were collected from living patients of the same age group admitted for surgical procedures 
Table 1: Light microscopic cellular changes according to time since death in oral mucosa samples $(\mathrm{N}=150)$

\begin{tabular}{|c|c|c|c|}
\hline \multirow{2}{*}{$\begin{array}{l}\text { Type of } \\
\text { tissue }\end{array}$} & \multicolumn{3}{|c|}{ PMI stage* } \\
\hline & Early & Intermediate & Late \\
\hline Epithelium & $\begin{array}{l}\text { - Cytoplasmic vacuolation (BM and GM) } \\
\text { - Homogenisation (BM and GM) } \\
\text { - Nuclear degeneration (BM and GM) } \\
\text { - Chromatin clumping (BM and GM) } \\
\text { - Arc-shaped nuclei (GM) }\end{array}$ & - Arc-shaped nuclei (BM) & $\begin{array}{l}\text { - Epithelial } \\
\text { shredding (BM) }\end{array}$ \\
\hline $\begin{array}{l}\text { Connective } \\
\text { tissue }\end{array}$ & - & $\begin{array}{l}\text { - Collagen lysis (BM) } \\
\text { - Melanin incontinency (BM and GM) } \\
\text { - Loss of epithelial connective tissue interface } \\
\text { (BM) } \\
\text { - Myofibril degeneration (BM) } \\
\text { - Mucous acini degeneration (BM) }\end{array}$ & $\begin{array}{l}\text { - RBC clumping } \\
\text { (BM and GM) } \\
\text {. Collagen lysis } \\
(\mathrm{GM})\end{array}$ \\
\hline
\end{tabular}

$P M I=$ post-mortem interval; $B M=$ buccal mucosa $G M=$ gingival mucosa $; R B C=$ red blood cell. ${ }^{*}$ PMI stage was categorised as early $(<12.5$ hours since death), intermediate (12.5-20.5 hours since death) or late ( $>20.5$ hours since death).

Table 2: Predictive potential of selected light microscopic cellular features to estimate post-mortem interval in oral mucosa samples* from recently deceased individuals $(\mathrm{N}=75)$

\begin{tabular}{|c|c|c|c|c|c|c|}
\hline \multirow[t]{2}{*}{ Type of tissue } & & \multirow[t]{2}{*}{ Feature } & \multicolumn{3}{|c|}{$\begin{array}{l}\text { Frequency according to PMI stage }{ }^{\dagger}, \\
\mathbf{n}(\%)\end{array}$} & \multirow[t]{2}{*}{$P$ value } \\
\hline & & & $\begin{array}{l}\text { Early } \\
(\mathbf{n}=25)\end{array}$ & $\begin{array}{l}\text { Intermediate } \\
\quad(\mathbf{n}=28)\end{array}$ & $\begin{array}{l}\text { Late } \\
(\mathrm{n}=22)\end{array}$ & \\
\hline \multirow[t]{5}{*}{ Epithelium } & GM & Homogenisation & $10(40)$ & $24(86)$ & $19(86)$ & 0.011 \\
\hline & $\mathrm{BM}$ & Nuclear degeneration & $18(72)$ & $26(93)$ & $21(95)$ & 0.021 \\
\hline & GM & Cytoplasmic vacuolation & $17(68)$ & $25(89)$ & $20(91)$ & 0.012 \\
\hline & $\mathrm{BM}$ & Chromatin clumping & $14(56)$ & $24(86)$ & $19(86)$ & 0.029 \\
\hline & $\mathrm{BM}$ & Arc-shaped nuclei & $6(24)$ & $19(68)$ & $13(59)$ & 0.038 \\
\hline \multirow[t]{6}{*}{ Lamina propria } & $\mathrm{BM}$ & Clumping and lysis of RBCs & $6(24)$ & $10(36)$ & $13(59)$ & 0.049 \\
\hline & GM & Clumping and lysis of RBCs & $2(8)$ & $8(29)$ & $9(41)$ & 0.010 \\
\hline & GM & Collagen lysis & $4(16)$ & $7(25)$ & $13(59)$ & $<0.001$ \\
\hline & $\mathrm{BM}$ & Myofibril degeneration & $2(8)$ & $8(29)$ & $6(27)$ & 0.003 \\
\hline & $\mathrm{BM}$ & Melanin incontinency & $7(28)$ & $15(54)$ & $17(77)$ & 0.017 \\
\hline & $\mathrm{BM}$ & Epithelial connective tissue separation & $0(0)$ & $6(21)$ & $4(18)$ & 0.028 \\
\hline Salivary gland & $\mathrm{BM}$ & Mucous acini degeneration & $9(36)$ & $15(54)$ & $16(73)$ & 0.013 \\
\hline
\end{tabular}

$P M I=$ post-mortem interval; $B M=$ buccal mucosa; $G M=$ gingival mucosa; $R B C s=$ red blood cells. "Including one sample each of BM and $G M$. ${ }^{\dagger} P M I$ stage was categorised as early ( $<12.5$ hours since death), intermediate (12.5-20.5 hours since death) or late ( $>20.5$ hours since death).

such as gingivectomy, surgical impaction and biopsies of reactive lesions.

The excised tissues were fixed in $10 \%$ buffered formalin. Subsequently, formalin-fixed paraffinembedded (FFPE) blocks were prepared for both post-mortem and antemortem samples. The FFPE blocks were sectioned into slides of $4 \mu \mathrm{m}$ in thickness and stained with routine haematoxylin and eosin (H\&E) stain. In addition, 21 slides were stained with van Gieson stain (Hi-Media Laboratories Pvt. Ltd., Einhausen, Germany) and 15 slides were stained with periodic acid-Schiff (PAS) stain (Hi-Media Laboratories Pvt. Ltd.) to determine changes in the intensity of collagen/reticulin fibres and basement membranes/mucous acini, respectively, with time since death.

Stained tissue sections were viewed under a light microscope to evaluate epithelial and connective tissue changes. These changes were categorised according to PMI stage as either early $(<12.5$ hours since death), intermediate (12.5-20.5 hours since death) or late (>20.5 hours since death). Epithelial changes included cytoplasmic vacuolation, changes to the cellular outline, homogenisation, changes to the nuclear outline, nuclear degeneration, nuclear vacuolation, arc-shaped nuclei, chromatin clumping and epithelial shredding (i.e. separation of layers of the epithelium). Connective tissue changes to the lamina 
propria included collagen lysis, fibroblast vacuolation, inflammation, homogenisation, clumping and lysis of the red blood cells (RBCs) and melanin incontinency. In addition, the buccal submucosa was examined for degeneration of the myofibrils and salivary glands. The interface between the epithelium and connective tissue was also studied. Changes in the intensity of van Gieson stains in the collagen fibres as well as that of PAS stains in the basement membrane and mucous acini were recorded via consensus. The same slide was evaluated consecutively by three different pathologists and interpreted subjectively as being either of mild, moderate or severe intensity.

Data were entered into an Excel spreadsheet, Version 2013 (Microsoft Corp., Redmond, Washington, USA), and analysed using the Statistical Package for the Social Sciences (SPSS), Version 24.0 (IBM Corp., Armonk, New York, USA). The antemortem and postmortem samples were compared for the frequency of microscopic changes in the epithelium, lamina propria and submucosa. Early, intermediate and late changes in post-mortem samples were identified depending upon a minimum frequency of $>40 \%$ of specific microscopic features at each PMI stage. Logistic regression analysis was conducted to determine the predictive efficiency of these parameters in the estimation of time since death. Changes in staining intensity of the collagen fibres, mucous acini and basement membrane for special stains were evaluated with respect to changes in PMI using analysis of variance.

Ethical approval for this study was obtained from the institutional ethical committee of the Institute of Dental Sciences, Siksha 'O' Anusandhan University (\#DMR/IMS-3H/SOA/180053). Written consent was obtained from the next of kin of the deceased individuals.

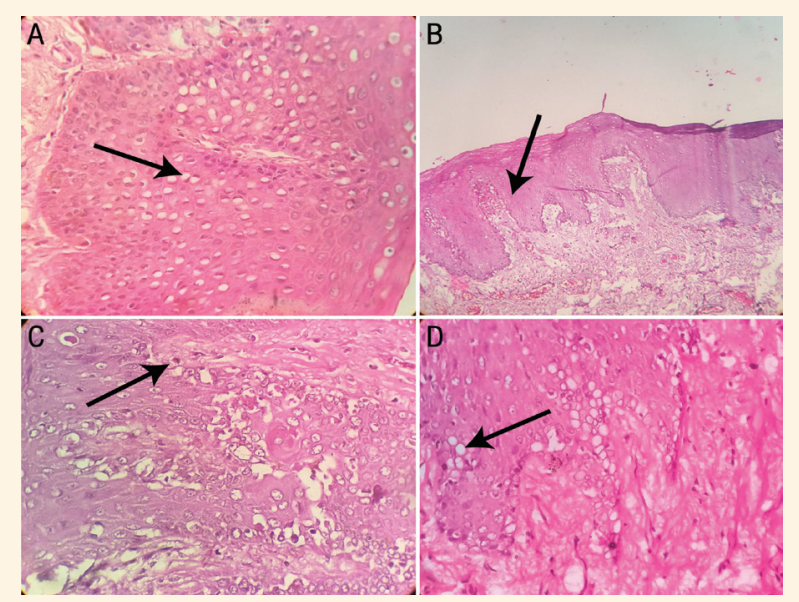

Figure 1:Haematoxylin and eosin stains at 10x magnification of post-mortem samples of oral mucosa epithelial cells showing (A) arc-shaped nuclei (arrow), (B) epithelial homogenisation (arrow), (C) nuclear degeneration (arrow) and (D) cytoplasmic vacuolation (arrow). These cellular and architectural microscopic changes were found to be significant predictors of post-mortem interval.

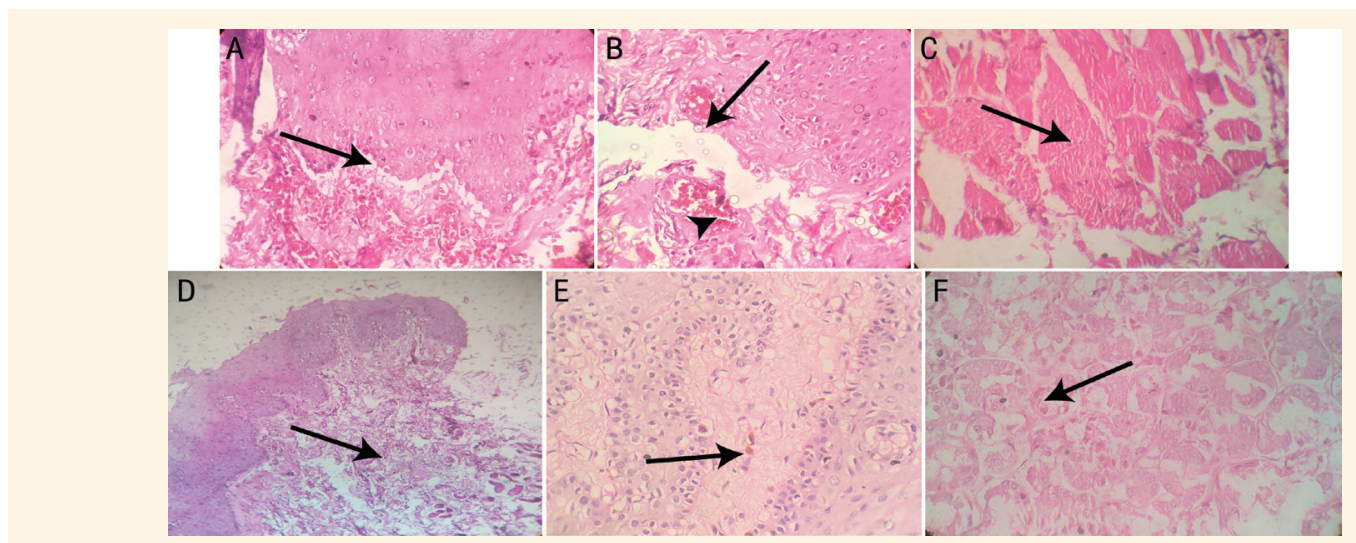

Figure 2: Haematoxylin and eosin stain at 10x magnification of post-mortem samples of oral mucosa connective tissue cells showing (A) epithelial connective tissue separation (arrow), (B) clumping (arrow) and lysis (arrowhead) of the red blood cells, (C) myofibril degeneration (arrow), (D) collagen lysis (arrow), (E) melanin incontinency (arrow) and (F) degeneration of the mucous acini (arrow). These cellular and architectural microscopic changes were found to be significant predictors of post-mortem interval. 

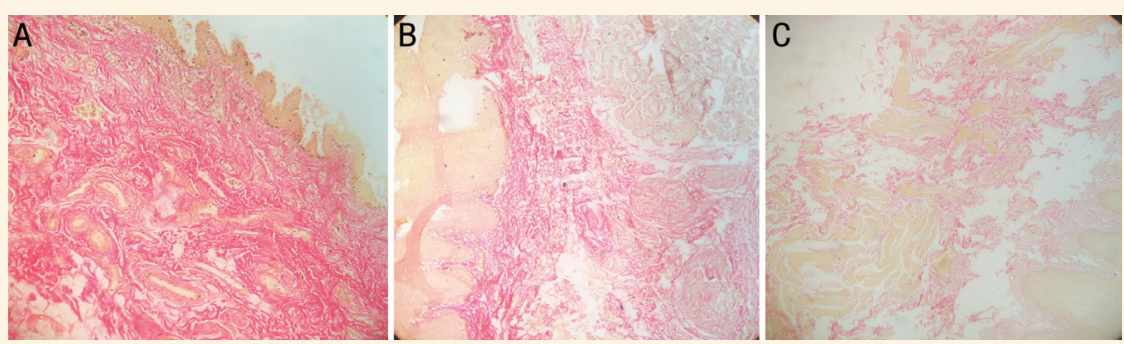

Figure 3: van Gieson stains at 10x magnification showing the (A) mild, (B) moderate and (C) severe decrease in staining intensity of collagen fibres in post-mortem samples of the oral mucosa as the post-mortem interval increased.

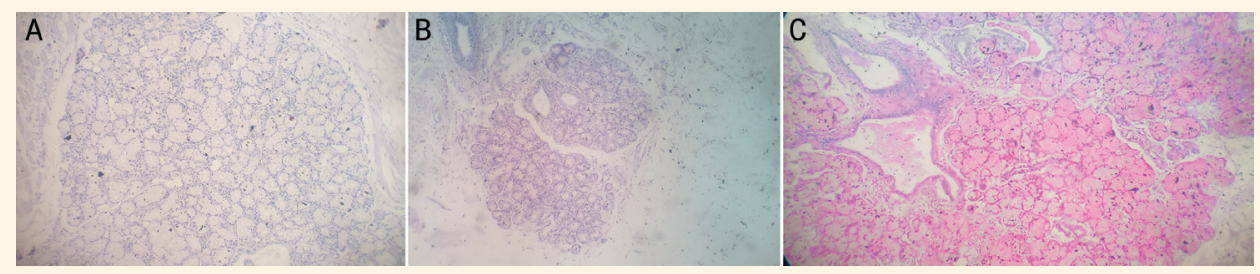

Figure 4: Periodic acid-Schiff stains at 10x magnification showing the (A) mild, (B) moderate and (C) severe increase in staining intensity of mucous acini in post-mortem samples of the oral mucosa as the post-mortem interval increased.

\section{Results}

A total of 150 oral mucosa samples comprising 75 gingival and 75 buccal mucosa samples were obtained from 75 deceased individuals. Of these, 51 (68\%) were male and $24(32 \%)$ were female. The most common cause of death was road traffic accidents $(n=37$; $49 \%$ ), followed by poisoning ( $\mathrm{n}=19 ; 25 \%)$, hypoxia $(\mathrm{n}=15 ; 20 \%)$ and cardiac arrhythmia $(\mathrm{n}=1 ; 1 \%)$. The reason for death in the remaining three patients was unknown. In terms of PMI stage, 25 (33\%) individuals had died < 12.5 hours previously, 28 (37\%) had died between $12.5-20.5$ hours previously and 22 (29\%) had died $>20.5$ hours previously.

In the epithelial cells, early microscopic changes seen within 12.5 hours of death included epithelial homogenisation, cytoplasmic vacuolation, nuclear degeneration and chromatin clumping with a progressive increase in the frequency of these features in the intermediate and late stages. Arc-shaped nuclei and epithelial shredding were intermediate and late changes, respectively. Interestingly, microscopic features of the connective tissue such as melanin incontinency and mucous acini degeneration were observed in the intermediate stage, whereas RBC clumping and lysis and collagen lysis were late changes. Epithelial connective tissue separation and myofibril degeneration were observed in $<40 \%$ of the cell population at all three PMI stages. In addition, inflammatory cells in the connective tissue stroma were observed in very few post-mortem tissues. Time-specific microscopic changes according to PMI stage are summarised in Table 1. The appearance of these features in the epithelium and connective tissue are shown in Figures
1 and 2, respectively. None of these architectural or cytological abnormalities were identified in the antemortem specimens.

In the buccal mucosa samples, a logistic regression analysis indicated that epithelial features such as nuclear degeneration, arc-shaped nuclei and chromatin clumping along with connective tissue features such as RBC clumping and lysis, melanin incontinency, degeneration of the myofibrils and salivary gland acini and epithelial connective tissue separation were significant predictors of PMI ( $P<0.050$ each). In turn, epithelial homogenisation, changes to the nuclear outline, nuclear vacuolation in the epithelial cells and collagen lysis and RBC clumping and lysis in connective tissue cells were significant predictors of PMI in the gingival samples $(P<0.050$ each) [Table 2 ].

There was a progressive decrease in staining intensity of collagen fibres in van Gieson stains [Figure 3]. The intensity of van Gieson stains in collagen fibres was found to be a significant predictor of time since death in both buccal mucosa and gingival samples $(P=$ 0.029). While there was no progressive change in PAS staining intensity in the basement membranes of either buccal mucosa or gingival samples as PMI increased, PAS staining intensity in salivary gland acini increased significantly with PMI $(P=0.021)$ [Figure 4].

\section{Discussion}

The current study was conducted on post-mortem samples of otherwise healthy buccal and gingival mucosae collected at three different intervals of time since death. The first objective was to identify 
differences between antemortem and post-mortem samples of oral mucosa among adults of similar age groups, while the secondary objective was to evaluate early, intermediate and late microscopic changes in oral tissues according to PMI stage. Finally, the study sought to determine the potential of these microscopic features in predicting time since death using both routine and special staining techniques.

With regards to the first objective, various degenerative microscopic features in the epithelium and connective tissue of the oral mucosa were observed in post-mortem samples, but not in antemortem samples. In particular, cytoplasmic vacuolation, epithelial homogenisation, nuclear degeneration and chromatin clumping were early changes in both the buccal and gingival mucosa. However, while arc-shaped nuclei were seen at $<12.5$ hours since death in the gingival mucosa, this feature was only seen at $\geq 12.5$ hours in the buccal mucosa. Similarly, melanin incontinency was the only microscopic change observed in both the buccal and gingival mucosa at $\geq 12.5$ hours, whereas collagen lysis, degeneration of the myofibrils and salivary gland acini and epithelial connective tissue separation were seen at this stage only in the buccal mucosa. These findings support those of previous research conducted by Pradeep et al. and Yadav et al. ${ }^{7,15}$

In addition, the present study also focused on additional features such as arc-shaped nuclei as an early change in the gingiva and as an intermediate change in the buccal mucosa. The identification of epithelial shredding as a late microscopic change in the buccal mucosa is in agreement with results reported by Yadav et al. in the gingival and labial mucosa. ${ }^{8,15}$ Microscopic changes in the connective tissue such as $\mathrm{RBC}$ clumping and lysis in both types of mucosae and collagen lysis in the gingiva occurred at $>20.5$ hours The latter finding supports previous research by Yadav et al. indicating that collagen disruption becomes increasingly apparent over time. ${ }^{8,15}$ However, Yadav et al. reported no abnormalities in the RBCs, a finding which contradicts that of the present study. ${ }^{7}$

The logistic regression analysis indicated that certain microscopic features in the epitheliumincluding homogenisation and cytoplasmic vacuolation in the gingiva and nuclear degeneration, arc-shaped nuclei and chromatin clumping in the buccal mucosawere significant predictors of PMI. Similarly, connective tissue features of RBC clumping and lysis, melanin incontinency, degeneration of the myofibrils and salivary gland acini and epithelial connective tissue separation in the buccal mucosa and RBC clumping and lysis and collagen lysis in the gingiva were significant PMI predictors. While the role of melanin incontinency in estimating PMI has been evidenced in the skin, no previous study had yet explored its potential in the oral mucosa. ${ }^{16}$

Several connective tissue stains are currently used in forensic pathology practice to order to identify the nature of death. ${ }^{17,18}$ However, to the best of the authors' knowledge, no other studies have sought to determine their role in predicting PMI. The current study found that, with an increase in time elapsed since death, there was a significant progressive decrease in the staining intensity of collagen fibres using van Gieson stains, whereas there was a significant progressive increase in the staining intensity of salivary gland acini using PAS stains. Therefore, either of these two staining techniques may be useful in the estimation of PMI. The van Gieson stain is a simple stain which tints collagen red and other tissues yellow and is often used for the differential demonstration of collagen. Varying shades of red are produced depending on the density of the collagen fibres. A progressive decrease in stain intensity can be attributed to collagen lysis, a feature observed in both the intermediate and late PMI stages; alternatively, this may be due to a decrease in the thickness of the submucosa or lamina propria. ${ }^{15,19}$

In a previous study, Mahalakshmi et al. evaluated histological changes in gingival tissue based on an evaluation of unfixed antemortem tissues alone. ${ }^{9}$ Yadav et al. also studied post-mortem changes in oral mucosa without comparison with antemortem mucosa. ${ }^{15}$ In comparison, other researchers have compared antemortem and post-mortem mucosal samples to determine post-mortem cellular changes. ${ }^{7,8,10,16}$ Three studies have been conducted to associate changes in salivary gland oral mucosa with time since death. ${ }^{11,12,20}$ Based on evaluations of the cellular changes in antemortem and post-mortem mucosa, Gururaj suggested that the decomposition process begins in the initial 24 hours, while Pradeep et al. concluded that the decomposition process at the cellular level begins within 10 hours of death.,21 Yadav et al. investigated 10 antemortem mucosa samples to determine nuclear and cytoplasmic changes at various time intervals and proposed the usefulness of histological changes in the oral mucosa for estimating PMI. ${ }^{8}$ In a recent study, Muthukrishnan et al. described early and late postmortem changes in five antemortem and five postmortem gingival samples. ${ }^{10}$

Due to sample size limitations, none of the aforementioned studies were able to provide statistical confirmation of the usefulness of light microscopic cellular changes in the oral mucosa as a method of predicting time since death. ${ }^{9-11,15,20,21}$ Moreover, several studies either evaluated post-mortem cellular changes in the gingiva alone or in the labial mucosa. ${ }^{7,8,15} \mathrm{No}$ studies have yet sought to assess the role of these 
changes in the buccal mucosa. Indeed, the present study is the first to establish the usefulness of this approach in both the gingiva and buccal mucosa, which are distinct types of oral mucosal tissues which are different from both an anatomical and structural perspective. For instance, the mean thickness of the epithelium in the buccal mucosa and gingiva are 294 $\pm 68 \mu \mathrm{m}$ and $239 \pm 57 \mu \mathrm{m}$, respectively. ${ }^{22}$ In addition, the thickness of the connective tissue also differs in these two zones as submucosa is absent in the gingiva, but present in the buccal mucosa. As such, characterisation of the role of the oral mucosa in identifying PMI requires the individual evaluation of different mucosal regions.

This study is subject to certain limitations, such as the absence of quantifying the cellular and nuclear changes to establish any association with PMI and observing the post-mortem cellular and nuclear changes in different environments. These factors would have led to further accuracy in determining PMI. However, the strengths of this study lie in the inclusion of a large sample size, the evaluation of two different kinds of oral mucosa tissue-including the buccal mucosa-and examination of the role of microscopic features in routine H\&E stains as well as special stains such as van Gieson and PAS. Based on these findings, further research is recommended to determine the point in time after death at which microscopic changes cease. In addition, advanced histological techniques, such as immunohistochemistry and electron microscopy, should also be investigated as other potential methods of determining post-mortem changes in the oral tissues.

\section{Conclusion}

Several microscopic features of the gingiva and buccal mucosa were found to be significant predictors of PMI including epithelial homogenisation, cytoplasmic vacuolation, nuclear degeneration, arc-shaped nuclei, chromatin clumping, RBC clumping and lysis, melanin incontinency, myofibril degeneration, salivary gland acini degeneration and epithelial connective tissue separation. In addition, there was a progressive decrease and increase in staining intensity with the use of van Gieson and PAS stains, respectively. To the best of the authors' knowledge, this study is the first of its kind to identify light microscopic features in two distinct kinds of oral mucosae using two special staining techniques in addition to conventional $\mathrm{H} \& \mathrm{E}$ staining.

\section{CONFLICT OF INTEREST}

The authors declare no conflicts of interest.

\section{FUNDING}

No funding was received for this study.

\section{References}

1. Buchan MJ, Anderson GS. Time since death: A review of the current status of methods used in the later post-mortem interval. Can Soc Forensic Sci 2001; 34:1-22. https://doi.org/10.1080/00 085030.2001 .10757514

2. Wang Q, Lin HC, Xu JR, Huang P, Wang ZY. Current research and prospects on post-mortem interval estimation. Fa Yi Xue Za Zhi 2018; 34:459-67. https://doi.org/10.12116/j.issn.10045619.2018.05.002.

3. Milroy CM. Forensic taphonomy: The post-mortem fate of human remains. BMJ 1999; 319:458. https://doi.org/10.1136/ bmj.319.7207.458.

4. Kumar V, Cotran RS, Robbins SL. Textbook of basic pathology, 5th ed. Elsevier, India, 1992, P 22-5.

5. Mel'nikov IuL. [Post-mortem changes in the liver as a criterion for the establishment of the time of death (experimental study)]. Sud Med Ekspert 1969; 12:20-3

6. Tomita Y, Nihira M, Ohno Y, Sato S. [Histological study of early post-mortem changes in various organs: Comparison of the paraffin embedding method and the epoxy resin embedding method]. Nihon Hoigaku Zasshi 1999; 53:207-17.

7. Pradeep GL, Uma K, Sharada P, Prakash N. Histological assessment of cellular changes in gingival epithelium in antemortem and post-mortem specimens. J Forensic Dent Sci 2009; 1:61-5. https://doi.org/10.4103/0974-2948.60375.

8. Yadav AB, Angadi PV, Kale AD, Yadav SK. Histological assessment of cellular changes in post-mortem gingival specimens for estimation of time since death. J Forensic Odontostomatol 2015; 33:19-26.

9. Mahalakshmi V, Gururaj N, Sathya R, Sabarinath TR, Sivapathasundharam B, Kalaiselvan S. Assessment of histological changes in antemortem gingival tissues fixed at various time intervals: A method of estimation of post-mortem interval. J Forensic Dent Sci 2016; 8:114. https://doi.org/10.4103/0975-1475.186373.

10. Muthukrishnan S, Narasimhan M, Paranthaman SK, Hari T, Viswanathan P, Rajan ST. Estimation of time since death based on light microscopic, electron microscopic, and electrolyte analysis in the gingival tissue. J Forensic Dent Sci 2018; 10:34-9. https://doi.org/10.4103/jfo.jfds_36_17.

11. Azevedo-Alanis LR, Tolentino Ede S, de Assis GF, Cestari TM, Lara VS, Damante JH. Acinar autolysis and mucous extravasation in human sublingual glands: A microscopic postmortem study. J Appl Oral Sci 2015; 23:459-66. https://doi. org/10.1590/1678-775720150139.

12. Agarwal S, Chaudhary M, Gawande M, Gupta P. Documentation of post-mortem changes in salivary gland architecture and staining characteristics. J Forensic Dent Sci 2016; 8:113. https:// doi.org/10.4103/0975-1475.186360.

13. Poór VS, Lukács D, Nagy T, Rácz E, Sipos K. The rate of RNA degradation in human dental pulp reveals post-mortem interval. Int J Legal Med 2016; 130:615-19. https://doi.org/10.1007/ s00414-015-1295-y.

14. Carrasco PA, Brizuela CI, Rodriguez IA, Muñoz S, Godoy ME, Inostroza $\mathrm{C}$. Histological transformations of the dental pulp as possible indicator of post mortem interval: A pilot study. Forensic Sci Int 2017; 279:251-7. https://doi.org/10.1016/j. forsciint.2017.09.001.

15. Yadav A, Angadi PV, Hallikerimath S, Kale A, Shetty A. Applicability of histologic post-mortem changes of labial mucosa in estimation of time of death - A preliminary study. Aust J Forensic Sci 2012; 44:343-52. https://doi.org/10.1080/0045061 8.2012 .674977 
16. Bardale RV, Tumram NK, Dixit PG, Deshmukh AY. Evaluation of histologic changes of the skin in post-mortem period. Am J Forensic Med Pathol 2012; 33:357-61. https://doi.org/10.1097/ PAF.0b013e31822c8f21.

17. Lau G, Lai SH. Forensic histopathology. In: Tsokos M (Ed) Forensic Pathology Reviews, volume 5. Totowa, New Jersey, USA:Humana Press, 2008.Pp. 239-65.https://doi.org/10.1007/9 78-1-59745-110-9 13.

18. Parai JL, Milroy CM. The utility and scope of forensic histopathology. Acad Forensic Pathol 2018; 8:426-51. https://doi. org/10.1177/1925362118797602.

19. Nahar N, Ara S, Zohora F, Siddika D, Yesmin T. Histological study on thickness of different layers of the gallbladder: A postmortem study. Bangladesh J Anat 2011; 9:41-4. https://doi. org/10.3329/bja.v9i1.8147.
20. Takeda Y. Oncocytic hyperplasia in the human minor salivary glands: A post-mortem study. Virchows Arch A Pathol Anat Histopathol 1993; 423:105-9. https://doi.org/10.1007/BF01606584.

21. Gururaj NS, Sivapathasundharam B. Post mortem findings in the gingiva assessed by histology and exfoliative cytology. J Oral Maxillofac Pathol 2004; 8:18-21.

22. Prestin S, Rothschild SI, Betz CS, Kraft M. Measurement of epithelial thickness within the oral cavity using optical coherence tomography. Head Neck 2012; 34:1777-81. https://doi.org/10.10 02/hed.22007. 